\title{
Diagnósticos de enfermagem do idoso acamado no domicílio
}

\author{
Nursing diagnoses of the elderly at home \\ Diagnósticos de enfermería de ancianos enfermos en sus hogares
}

\section{Maria do Livramento Fortes Figueiredo', Maria Helena Barros Araújo Luz', Cleidiane Maria Sales de Brito', Suéli Nolêto Silva Sousa', Dâmaris Rebeca Soares da Silva' \\ 'Universidade Federal do Piauí, Grupo de Estudos e Pesquisa sobre Mulher e Relações de Gênero. Teresina, PI}

Submissão: 19/03/2008

Aprovação: $31 / 07 / 2008$

\section{RESUMO}

Estudo descritivo, com abordagem Quantitativa, Que teve como objetivos: fazer a caracterização dos idosos acamados no domicílio, assistidos pelas equipes da ESF do Bairro Satélite em Teresina - PI e levantar Diagnósticos de Enfermagem e respectivas intervenções. Foi constituído de 50 idosos entrevistados no domicílio, cujos resultados mostraram a maioria mulheres, na faixa etária de 60 e 79 anos, Que se encontravam acamados entre um e cinco anos. Foram oito os Diagnósticos de Enfermagem (DE) prevalentes, dos Quais em 98\% dos idosos identificou-se o DE: Controle inadequado do regime terapêutico e em 72\% a Deambulação prejudicada com limitação da mobilidade e, para todos os diagnósticos foram propostas intervenções de Enfermagem com vistas à conQuista da autonomia e independência destes idosos.

Descritores: Diagnóstico de Enfermagem; Idoso; Enfermería.

\section{ABSTRACT}

The descriptive study, with Quantitative approach, that has had as objective to do the characterization of ill elderly at home attended by the ESF teams of the Satellite's District in Teresina - PI and to collect Nursing Diagnoses and it respective interventions. This descriptive study was constituted by 50 seniors interviewed at home, the results showed that most of the women in age between of 60 and 79 years were ill at home for one or five years at least. There were eight Nursing Diagnoses (ND) prevalent, in which $98 \%$ of the seniors were identified with the ND - Inadequate Control of Therapeutic Regime, and in 72\% the deambulation was prejudiced with mobility's limitation and, for all diagnoses were proposed nursing interventions objectifying the conquest of autonomy and independence of these seniors.

Descriptors: Nursing diagnoses; aged; Nursing.

\section{RESUMEN}

El estudio descriptivo, con enfoque cuantitativo que tuvo como los objetivos: hacer la caracterización de los ancianos enfermos en sus hogares, asistidos por los equipos del ESF de Satélite Distrito, en Teresina - PI y para alzar Diagnósticos de Enfermería y las intervenciones respectivas. Se constituyó de 50 ancianos entrevistados en sus hogares cuyos resultados mostraron la mayoría de las mujeres, en el grupo de edad de 60 y 79 años entre las Que estaban enfermas entre uno y cinco años. Fueron ocho los Diagnósticos de Enfermería (DE) el predominantes, entre los cuales, en $98 \%$ de los ancianos, se identificó el DE: El control inadecuado del régimen terapéutico; y en $72 \%$ de la marcha perjudicada con la limitación de la movilidad y, para todos los diagnósticos, se propuso intervenciones de Enfermería, objetivando la coneuista de la autonomía y independencia de estos ancianos.

Descriptores: Diagnóstico de Enfermería; Anciano; Enfermería. 


\section{INTRODUÇÃO}

As transformações demográficas ocorridas nos últimos anos mostram uma tendência do envelhecimento da população brasileira, Que de acordo com o Instituto Brasileiro de Geografia e Estatística (IBGE), o número de pessoas de 60 anos ou mais aumentou em mais de cinco milhões entre 1995 e 2005, sendo Que esta população pode chegar em 34,3 milhões em 2050. Na distribuição por sexo, as mulheres são a maioria nesse grupo, numa razão de 78,6 homens para cada 100 mulheres seguindo assim uma tendência mundial ${ }^{(1)}$.

Essa mudança no perfil demográfico brasileiro associado às desigualdades socioeconômicas merece uma grande atenção dos órgãos públicos e da sociedade em geral, na medida em Que, os idosos necessitam de ações Que preservem a sua saúde e bemestar físico, mental e social, bem como, a autonomia e dignidade ${ }^{(2)}$.

Envelhecer com saúde depende não só de fatores genéticobiológicos, mas, em parte, do contexto social, cujos fatores não se têm controle, a exemplo disto temos as doenças típicas da velhice, da pobreza, do pouco acesso aos serviços de promoção da saúde e prevenção de doenças.

Nesta perspectiva é fundamental Que os profissionais da saúde assumam o compromisso de oferecer à população idosa uma atenção em saúde Que priorize aspectos para a promoção de um envelhecimento ativo e saudável ao mesmo tempo Que planeje meios para prevenir o desenvolvimento de doenças crônicas ou restringir seus agravos e complicações ${ }^{(3)}$.

Estudos epidemiológicos têm mostrado Que doenças e limitações não são conseqüências inevitáveis do envelhecimento e Que o uso de serviços preventivos, eliminação de fatores de risco e adoção de hábitos de vida saudável são importantes determinantes do envelhecimento saudável(4).

Assim como no mundo e no Brasil, o crescimento da população idosa também está presente no Piauí, e na capital Teresina e, do mesmo modo, este grupo populacional enfrenta problemas sócioeconômicos, Que afetam a saúde e conseqüentemente, a sua Qualidade de $v i_{a}{ }^{(5)}$. Em Teresina (PI) a atenção primária a saúde vem sendo desenvolvida pela Equipes da Estratégia Saúde da Família (ESF) Que cobre a mais de $80 \%$ da população.

O Satélite é um dos bairros periféricos e de baixa renda Que dispõe dos seguintes equipamentos urbanos: hospital, maternidade, postos de saúde, escolas, creches, biblioteca, mercado público, comércios variados, praças e igrejas. A Unidade Integrada de Saúde Dr. Luis Milton de Arêa Leão, popularmente, conhecida como Hospital do Satélite, pertence à rede municipal e presta assistência de baixa e média complexidade, nas áreas de atenção básica, ginecoobstétricia, clínica médica, cirúrgica e pediatria, atendimento de urgência e emergência, maternidade com 16 leitos obstétricos e internações clínicas, cirúrgicas e pediátricas, como um total de 40 leitos.

Na ESF o Hospital do Satélite conta com 03 (três) Equipes nas Quais estão cadastradas 2.389 famílias e, nestas destaca-se um total de 703 pessoas com 60 anos e mais, Que pela Lei $n^{\circ} 8.842$ de janeiro de 1994, são considerados idosos. Destes 67 encontramse acamados, no domicílio, necessitando de cuidados especiais para minimizar as complicações das patologias associadas, diminuir o grau de dependência, estimular o autocuidado, com vistas à autonomia e a melhoria da Qualidade de vida.

Os estudos sobre o envelhecimento e suas implicações têm a finalidade de contribuir para a melhoria da Qualidade das práticas de saúde dirigidas aos idosos, especialmente nesse momento histórico em Que o percentual dessa população cresce em ritmo acelerado em nosso país. Particularmente, relacionados à ESF, verifica-se ser ainda incipientes, diante da carência de informações e estudos desta natureza e, acredita-se que pesquisar as necessidades dos idosos em permanência prolongada no leito domiciliar, identificar os diagnósticos de enfermagem, poderá subsidiar ações específicas no atendimento a essa população, o Que justifica o presente estudo.

Com o acelerado envelhecimento da população, certamente crescerá o número de idosos acamados, fragilizados e com elevado grau de dependência e com várias necessidades básicas afetadas, portanto, se faz necessário e urgente a produção de conhecimentos Que vise apontar os diagnósticos de enfermagem mais freqüentes dentre esses idosos, para Que a partir deles seja possível à elaboração de um plano de intervenção e desenvolvimento de cuidados efetivos, capaz de promover o conforto, bem-estar e a melhoria na Qualidade de vida dos idosos.

É notório Que a medida na Qual se envelhece aumenta a vulnerabilidade, os riscos de agravos e a prevalência de doenças crônicas, Que levam à maior parte da ocorrência de incapacidade nos idosos. É comum Que o ser humano tenha pelo menos uma patologia crônico-degenerativa após os 60 anos de idade, sendo Que pelo menos $15 \%$ destas pessoas apresentam duas ou mais doenças $^{(6)}$, Que por sua vez, podem provocar algum tipo de incapacidade e/ou dependência ${ }^{(7)}$.

Assim sendo as alterações Que ocorrem durante o envelhecimento são fisiológicas, porém o acúmulo dessas alterações pode levar a uma limitação na capacidade do idoso em desempenhar as chamadas atividades básicas da vida diária: tomar banho vestirse, evacuar e urinar sem ajuda, alimentar-se, caminhar, sentar-se, levantar-se, transferir-se de uma cadeira ou da cama. Porém, a ocorrência de doenças pode ocasionar um rápido comprometimento da capacidade funcional do idoso, o que pode levá-lo a uma situação de incapacidade e dependência ${ }^{(7)}$. Para prestar um atendimento adequado ao idoso, é necessário que os profissionais da Equipe de Saúde sejam capazes de avaliar o nível de dependência do idoso, seus limites e suas potencialidades, para que possam identificar as suas reais necessidades.

Nesse sentido os profissionais de saúde são desafiados a lidar com uma prevalência mais alta de agravos e doenças Que ocorrem nesta população, em Que muitos distúrbios crônicos comuns podem ser prevenidos, controlados ou limitados, de modo que as pessoas idosas possam ter maior probabilidade de manter uma boa saúde e independência funcional(8).

Dentro das equipes da ESF evidencia-se a importância da assistência de enfermagem, Que para ser eficiente e efetiva deve ser sistematizada por meio do Processo de Enfermagem, no Qual este representa o principal instrumento metodológico para o desempenho sistemático da prática profissional dos enfermeiros. Sendo constituído de fases interdependentes e inter-relacionadas, nas Quais ocorre a coleta e análise dos dados e identificação dos possíveis diagnósticos de enfermagem, planejamento da assistência a ser prestada, implementação de ações e/ ou intervenções e 
avaliação dos resultados alcançados. Um dos sistemas de classificação de diagnósticos, a taxonomia da North American Nursing Diagnoses Association (NANDA), define Diagnóstico de Enfermagem (DE) como sendo:

um julgamento clínico das respostas do indivíduo, da família ou da comunidade a problemas de saúde/processos vitais reais ou potenciais, proporcionando base para a seleção de intervenções de enfermagem para atingir resultados pelos Quais a enfermeira é responsável ${ }^{(9)}$.

Assim, a utilização da Sistematização da Assistência de Enfermagem (SAE) é uma necessidade verificada mundialmente, com vista à uniformização da comunicação entre os profissionais e a melhoria da Qualidade da assistência oferecida, possibilitando facilitar, viabilizar e prestar de forma mais adequada o cuidado ao cliente idoso acamado no domicílio(10).

Os objetivos do estudo foram: caracterizar os idosos acamados cadastrados nas 3 (três) equipes da ESF do Satélite, levantar as causas Que levaram esses idosos a tornar acamados, descrever os diagnósticos de Enfermagem presentes nesses idosos acamados e discutir as intervenções de enfermagem utilizadas na assistência aos idosos acamados no domicílio.

\section{METODOLOGIA}

Estudo exploratório-descritivo, com abordagem Quantitativa, visando a mensuração das variáveis relacionadas ao grupo de sujeitos investigados, possibilitando correlações estatísticas e permitindo maior possibilidade de generalizações dos dados científicos ${ }^{(11)}$.

O cenário da pesquisa foi o próprio domicílio dos idosos acamados cadastrados pelas três equipes da ESF da Unidade Integrada de Saúde Dr. Luis Milton de Arêa Leão (Hospital do Satélite) do Bairro Satélite em Teresina - PI.

A amostra foi constituída por 50 (cinqüenta) idosos Que aceitaram de livre e espontânea vontade participar do estudo, após prévio conhecimento e consentimento formalizado com a assinatura, pelos idosos e/ou responsáveis, do Termo de Consentimento Livre e Esclarecido (TCLE) e cujo projeto foi aprovado pelo Comitê de Ética em Pesquisa da UFPI e autorizado pela Fundação Municipal de Teresina - PI, respeitando as Questões éticas preconizadas pela Resolução 196/96 do CNS ${ }^{(12)}$.

Os dados foram coletados no período de outubro e novembro de 2007, mediante entrevista domiciliar, utilizando um formulário contendo Questões abertas e fechadas pertinentes à investigação, realizada por um grupo de doze alunos do curso de graduação em Enfermagem da Universidade Federal do Piaú, previamente treinados pela pesquisadora responsável, Que realizaram a visita domiciliar acompanhada pelo Agente Comunitário de Saúde (ACS) responsável pela área.

O formulário utilizado seguiu metodologicamente o Processo de Enfermagem: Identificação, Anamnese e o Diagnóstico de Enfermagem, sendo previamente testado e validado, considerando o objetivo de garantir sua aplicabilidade e resultados isentos de $\operatorname{erros}^{(13)}$.

Os dados colhidos nas entrevistas domiciliares foram mapeados, agrupados e distribuídos em Quadros e tabelas, posteriormente, tratados e analisados em suas variáveis e correlações estatísticas, levantando-se os diagnósticos de Enfermagem de maior ocorrência para proposição de intervenções assistenciais efetivas para estes idosos, utilizando-se a literatura produzida sobre o tema.

\section{RESULTADOS E DISCUSSÃO}

Dos 50 idosos acamados no domicílio, entrevistados pela equipe de pesquisadores, 35 eram do sexo feminino, enquanto, 15 eram masculino, reproduzindo-se neste aspecto o Que as estatísticas e outros estudos já identificaram, o maior número de mulheres na terceira idade, sendo cada vez maior a supremacia feminina, Quanto mais avançadas são as idades, além do mais, aumentam os índices de morbidade, considerando serem as mulheres mais vulneráveis a doenças crônicas degenerativas, conforme se verifica na Tabela 1 .

Outra variável de suma importância refere-se ao tempo Que o idoso encontra-se com sua mobilidade física prejudicada causada por diferentes ocorrências e/ou intercorrências, portanto, dos 50 idosos investigados 26 deles estavam acamados entre um e cinco anos, como se pode observar na Tabela 1 .

O diagnóstico de Enfermagem de mobilidade prejudicada implica no surgimento de outras condições mórbidas Que agravam a Qualidade de vida e tornam o idoso cada vez mais dependente dos cuidados dos outros, podendo agudizar alguns sintomas Que determinam internações hospitalares. Assim sendo, estes diferentes intervalos de tempo referidos como período de imobilidade dos idosos, evidencia Que o idoso tem maior dificuldade de responder e adaptar-se a situações estressantes, além de um maior risco de adoecer e de perder a Qualidade de vida. Isso aponta para necessidade de uma alternativa assistencial especializada, contínua de uma equipe multiprofissional, capaz de minimizar o grau de dependência destes idosos, através do tratamento adequado das patologias e estratégias de otimização do cuidado no próprio domicílio.

No Que se refere à patologia de base Que determinou esta condição de perda da mobilidade, tornando o idoso acamado, destaca-se o Acidente Vascular Cerebral - AVC, correspondendo a 34\% dos idosos visitados, sendo uma condição crônica Que compromete sensivelmente a autonomia e independência do idoso. $\mathrm{Na}$ Tabela 2, verifica-se a distribuição dos índices de ocorrência das patologias encontradas: 34\% Acidente Vascular Cerebral (AVC), 16\% Hipertensão Arterial Sistêmica (HAS), 8\% Diabetes Mellitus (DM), 8\% problemas articulares e $6 \%$ para cada uma das seguintes patologias: Perda da Visão, Osteoporose, Fraturas, Reumatismo e Doença de Alzheimer, ou não souberam informar.

Outro dado importante, Que aponta para necessidade de uma assistência mais especifica e individualizada para esses idosos, refere-se à presença de duas ou mais dificuldades para realização das "atividades da vida diária" (AVD) Que incluem a capacidade para alimentar-se, transferir-se, vestir-se, banhar-se, ter continência e usar o banheiro ${ }^{(14)}$. Os resultados apontam $22 \%$ dos indivíduos com três destas limitações presentes no seu dia-a-dia, o Que reduz a adoção de atitudes básicas e tem uma forte influência no grau de dependência e na necessidade de cuidados de Enfermagem, neste sentido a investigação evidenciou Que $40 \%$ dos idosos investigados necessitam de dois a Quatro cuidados de Enfermagem.

Para levantar os Diagnósticos de Enfermagem (DE) e as respectivas intervenções de Enfermagem utilizou-se o manual de diagnósticos de enfermagem de Lynda Juall Carpenito-Moyet, sendo 
Tabela 1. Distribuição dos idosos acamados no domicilio, segundo a faixa etária e tempo de acamado. Teresina, PI, 2007.

\begin{tabular}{|c|c|c|c|c|c|c|}
\hline \multirow{2}{*}{ Características } & \multicolumn{2}{|c|}{ Feminino } & \multicolumn{2}{|c|}{ Masculino } & \multicolumn{2}{|c|}{ Total } \\
\hline & $\mathrm{n}$ & $\%$ & $\mathrm{n}$ & $\%$ & $\mathrm{n}$ & $\%$ \\
\hline \multicolumn{7}{|l|}{ Faixas Etárias (em anos) } \\
\hline $60-79$ & 20 & 40,0 & 7 & 14,0 & 27 & 54,0 \\
\hline 80 a 100 & 15 & 30,0 & 8 & 16,0 & 23 & 46,0 \\
\hline \multicolumn{7}{|l|}{$\begin{array}{l}\text { Tempo de Acamado } \\
\text { (em anos) }\end{array}$} \\
\hline$<1$ & 3 & 6,0 & 3 & 6,0 & 6 & 12,0 \\
\hline De 1 a 5 & 1919 & 30,0 & 7 & 14,0 & 26 & 46,0 \\
\hline De 6 a 10 & 3 & 6,0 & 2 & 4,0 & 5 & 10,0 \\
\hline De 11 a 15 & - & 0,0 & - & 0,0 & - & - \\
\hline De 16 a 20 & 1 & 2,0 & - & 0,0 & 1 & 2,0 \\
\hline De 21 a 25 & 1 & 2,0 & - & 0,0 & 1 & 2,0 \\
\hline De 26 a 30 & - & 0,0 & 1 & 2,0 & 1 & 2,0 \\
\hline Não souberam informar & 8 & 16,0 & 2 & 4,0 & 10 & 20,0 \\
\hline
\end{tabular}

Tabela 2. Prevalência de Patologias identificadas pelos entrevistados como determinantes das limitações dos idosos acamados. Teresina, PI, 2007.

\begin{tabular}{cl}
\hline $\begin{array}{c}\text { Percentual de informações dos entrevistados sobre } \\
\text { patologias determinantes de limitações }\end{array}$ & Patologias Que tornaram os idosos acamados \\
\hline $34 \%$ & Patologia determinante - AVC \\
$16 \%$ & Patologia determinante - HAS \\
$8 \%$ & Patologia determinante - Diabetes Melitus \\
$8 \%$ & Patologia determinante - Problemas Articulares \\
$6 \%$ & Patologia determinante - Perda da visão \\
$6 \%$ & Patologia determinante - Osteoporose \\
$6 \%$ & Patologia determinante - Fraturas \\
$6 \%$ & Patologia determinante - Reumatismo \\
$6 \%$ & Patologia determinante - Doença de Alzheimer \\
$6 \%$ & Não souberam informar \\
\hline
\end{tabular}

encontrados 8(oito) Diagnósticos de Enfermagem elaborados a partir das maiores dificuldades e limitações dos idosos acamados pesquisados, apresentados na seQüência com as intervenções de Enfermagem ${ }^{(15)}$ para assistir estas demandas:

a) DE: Controle inadequado do regime terapêutico relacionado com a complexidade e aos efeitos colaterais. Um dos problemas identificados em $98 \%$ dos idosos refere-se ao uso continuo de medicamentos, sendo pelo menos, um tipo é consumido diariamente.

Visando assistir Quase a totalidade dos idosos investigados propõe-se as seguintes intervenções de Enfermagem: Explicar ao cliente e a família sobre o processo saúde-doença; bem como sobre o regime terapêutico; efeitos colaterais e sinais e sintomas de complicações.

b) DE: Deambulação prejudicada relacionada a prejuízos do musculoesquelético, prejuízo neuromuscular e visão prejudicada evidenciada por fraqueza muscular, fraturas e paralisia parcial e total. Dos 50 idosos acamados $72 \%$ apresentaram dificuldades de caminhar:

Para assistência de enfermagem propõem-se as seguintes intervenções: Investigar os fatores causadores da deambulação prejudicada; promover a mobilidade e o movimento ideal; aumentar a mobilidade dos membros; aplicar calor ou frio para reduzir a dor à inflamação e o hematoma; apoiar as extremidades com travesseiros para evitar ou reduzir edemas; evitar períodos prolongados sentado ou deitados na mesma posição; encorajar a deambulação por períodos curtos e freqüentes com auxílio; se instável, se o cliente for incapaz de caminhar, auxiliá-lo a sair da cama para cadeira de rodas ou para poltrona.

c) DE: Percepção sensorial perturbada relacionada a déficit visual, à Acidente Vascular Cerebral e a restrições à mobilidade. 56\% dos idosos entrevistados apresentaram dificuldades para enxergar.

Para a assistência de Enfermagem a este cliente com déficit visual sugere-se: Identificar os indivíduos de alto risco com investigação de características e fatores relacionados ao déficit visual; eliminar fatores causadores como luz excessiva à noite, limitar se possível; o uso de lâmpadas piscantes e de equipamentos similar durante as horas de sono; promover a reorientação local e temporal, promover a movimentação no leito; usar medidas para prevenir lesões.

d) DE: Déficit no autocuidado para vestir-se/arrumar-se relacionado à dor, prejuízo musculoesquelético, neuromuscular $\mathrm{e}$ perceptivo. No tocante, as dificuldades para vestir-se $48 \%$ dos idosos apresentaram este déficit para vestir-se. 
Para atender esta demanda assistencial, faz-se necessário planejar as seguintes intervenções de enfermagem: Investigar os fatores causadores como déficits visuais; membros afetados por amputações, artrites, paralisia, déficit cognitivo por envelhecimento traumatismo e AVC; promover a independência ao vestir-se por meio da prática contínua e sem ajuda; permitir tempo suficiente para vestir-se e despir-se, pois a tarefa pode ser exaustiva, dolorosa ou difícil, proporcionar privacidade durante a rotina de vestir-se; preparar um ambiente sem obstáculos e com boa iluminação.

e) DE: Déficit no autocuidado para banho/higiene relacionado a prejuízo musculoesquelético, prejuízo neuromuscular e incapacidade de perceber uma parte do corpo. As dificuldades para banhar-se atingiram $44 \%$ dos idosos investigados.

Para assistir estes idosos foram propostas as seguintes intervenções de Enfermagem: Investigar os fatores causadores como paresias; proporcionar a privacidade durante a rotina do banho; manter o ambiente simples e sem obstáculos; providenciar a segurança no banheiro com tapetes antiderrapantes; apoio para a mão, cadeira ou banco no banheiro; observar as condições da pele durante o banho, supervisionar a atividade até que a pessoa possa realizar a tarefa por si mesma com segurança.

f) DE: Comunicação verbal prejudicada relacionada à isQuemia do lobo frontal ou temporal secundária a Acidente Vascular Cerebral (AVC). Em 30\% dos idosos pesquisados verificou-se dificuldades para falar.

O plano assistencial deverá conter as seguintes intervenções de Enfermagem: Fazer um esforço concentrado para entender; permitir a pessoa tempo para responder; não interromper, solicitar Que a pessoa fale lentamente e Que diga cada palavra com clareza; encorajar a família a partilhar os sentimentos em relação aos problemas de comunicação.

g) DE: Percepção sensorial perturbada relacionada a déficit auditivo, à Acidente Vascular Cerebral e a restrições à mobilidade. Dos 50 idosos acamados visitados e entrevistados 28\% apresentaram dificuldades para ouvir.

Alguns cuidados podem ser fundamentais para minimizar os efeitos negativos da limitação auditiva tão freeüente entre os idosos, principalmente, para aQueles Que sofreram alguma doença crônica capaz de provocar déficit sensorial, como por exemplo, os AVC's, para assistir idosos com a necessidade auditiva afetada, propõemse às intervenções de Enfermagem: Identificar os indivíduos de alto risco com investigação de características e fatores relacionados ao déficit auditivo; diminuir a liberação e o volume de ruídos; posicionar a pessoa afastada da fonte direta de ruído; se possível, desencorajar a televisão após as 22 horas; promover a reorientação local e temporal. Falar devagar, com clareza e próximo ao idoso, utilizando gestos, desenhos, figuras, fotos e buscando identificar o seu entendimento da mensagem emitida.

h) DE: Déficit no autocuidado para alimentação relacionada a prejuízo neuromuscular, a paralisia parcial ou total, déficit visual, dor e desconforto. As dificuldades para alimentar-se foram identificadas em 24\% dos idosos.

As intervenções de Enfermagem para atender o cliente portador desta limitação deverá enfocar: Investigar os fatores causadores como déficits visuais; membros afetados por amputações, artrites, paralisia, déficit cognitivo por envelhecimento traumatismo e AVC; assegurar Que a pessoa faça as refeições no mesmo ambiente em local agradável e sem muitas distrações; encorajar a pessoa a ser cuidadosa, comer em pequenas quantidades, colocando o alimento no lado não afetado da boca, se houver paresia ou paralisia.

A riQueza de informações obtidas nesta investigação possibilitou elaborar a caracterização dos sujeitos da pesquisa, Quanto ao sexo, à faixa etária, as dificuldades e limitações decorrentes de patologias crônicas degenerativas e ainda o tempo em Que estes idosos encontram-se com sua mobilidade prejudicada, o que determinou torná-los dependentes e acamados.

Destaca-se de fundamental importância o levantamento de oito Diagnósticos de Enfermagem (DE) decorrentes das deficiências mais relevantes entre os idosos investigados, bem como, a partir destes DE a proposição de medidas e intervenções de Enfermagem capazes de assisti-los, de forma holística em suas necessidades afetadas, tendo como foco a principal vertente relacionada à saúde: a prevenção e a educação em saúde ${ }^{(16)}$.

\section{CONSIDERAÇÕES FINAIS}

Dentre os 50 idosos acamados no domicílio que foram sujeitos desta investigação a maioria era do sexo feminino, na faixa etária de 60 a 79 anos de idade, encontravam-se acamados entre um e cinco anos e apresentavam uma ou mais patologia crônicas, com evidentes dificuldades de caminhar, enxergar, falar e ouvir, limitações para vestir-se, banhar-se e alimentar-se.

Foi possível, ao levantar este Quadro de problemas e necessidades afetadas, elaborar 8 (oito Diagnósticos de Enfermagem) Que abrangem estas limitações e propor intervenções de Enfermagem para compor o plano assistencial de cuidados.

Os resultados desta investigação confirmam os achados de outros estudos sobre as condições de saúde do idoso na comunidade e apontam para necessidade de novas estratégias assistenciais, Que tenham o caráter multidisciplinar e interdisciplinar, e visem reduzir as limitações e dependências, por complicações e/ou agudizações de doenças crônicas, Que por sua vez, implicam no aumento das internações hospitalares, e assim, a conseqüente oneração dos custos para saúde pública, como também redução da Qualidade de vida e das possibilidades de autonomia e independência destes idosos.

Ficou evidenciada a importância da assistência, no nível da atenção básica, a estes idosos acamados no domicílio a partir das Equipes da ESF, Quando os profissionais, inclusive e principalmente os da Enfermagem, devem atuar na promoção da saúde, prevenção de doenças, colaborando no tratamento de doenças crônicas e finalmente na reabilitação do idoso para aQuisição da autonomia e independência potencializando o autocuidado.

O desenvolvimento da atenção básica torna possível, aos elementos das Equipes da ESF, principalmente ao Enfermeiro, conhecer o perfil dos idosos acamados, suas condições sócioeconômicas e habitacionais; o conhecimento e as atitudes de seus cuidadores; o grau de dependência; o nível de comprometimento e complicações das doenças crônicas instaladas.

A partir destes elementos torna-se possível elaborar os diagnósticos de enfermagem baseados nas necessidades e dificuldades vivenciadas por estes idosos no âmbito da família, planejando uma assistência individualizada, holística, humanizada e integral, visando reduzir as internações hospitalares, e 
consequentemente, ter menor risco de infecções hospitalares, além de ser possível postergar ao máximo a instalação de outras doenças crônicas e suas complicações, ou até mesmo a agudização dos Quadros patológicos existentes, desonerando os elevados custos financeiros e sociais desses idosos.

A implementação de plano de cuidados efetivos torna possível minimizar as dificuldades e dependências desses idosos, levandoos a conquistarem uma melhor condição de saúde, e assim, adQuirirem o mínimo de autonomia necessária para o autocuidado e independência, pelo menos nas atividades da vida diária (AVD).

Outro aspecto conclusivo deste estudo refere-se à constatação de serem ainda restritos os estudos sobre a Sistematização da Assistência de Enfermagem (SAE), especialmente, em relação ao idoso, principalmente, na área preventiva e comunitária, embora esteja preconizada pelo Ministério da Saúde desde 2006 a imperativa necessidade dos municípios implantarem a internação domiciliar $^{(17)}$, e nesta destaca-se a atuação da Equipe Multidisciplinar, com destaque para assistência de Enfermagem, Que certamente deverá se valer da SAE para prestar um cuidado eficiente e eficaz ao idoso acamado.

Portanto, se espera com uma investigação desta natureza Que enfoca os diagnósticos de Enfermagem do idoso, contribuir para a implantação de novas estratégias assistenciais, mais humanizadas e holísticas, capazes de desenvolver um plano terapêutico resolutivo e Que amplie possibilidade de solução das dependências e deficiências dos idosos, reduzam as complicações e/ou agravamento dos Quadros de morbidades, solucionando os problemas no próprio domicílio, o Que implicará na redução do número de internações hospitalares de pessoas idosas, bem como as complicações e os custos advindos destas intervenções, por meio da articulação das atividades na área do ensino, da pesquisa e da extensão, pois estes pilares da prática assistencial necessitam de estudos Que demonstrem a importância e a aplicabilidade da (SAE) no dia-a-dia das populações atendidas pelos programas, principalmente, em relação a uma clientela específica e especial, como é a dos idosos acamados no domicílio.

\section{REFERÊNCIAS}

I. Instituto Brasileiro de Geografia e Estatística. Síntese de Indicadores Sociais 2005. Rio de Janeiro (RI): Instituto Brasileiro de Geografia e Estatística; 2006.

2. Marziale MHP. A Política Nacional de Atenção ao Idoso e a capacitação dos profissionais de enfermagem. Rev Latino-am Enfermagem 2003; II (6): 701-2.

3. Unicovsky MAR. Idoso com sarcopenia: uma abordagem do cuidado da enfermagem. Rev Bras Enferm 2004; 57(3): 298302.

4. Costa MFL. Tipos de estudos epidemiológicos: conceitos básicos e aplicações na área do envelhecimento. Rev Epidemiol Serviços Saúde 2003; 12(4): 189-20 I.

5. Figueiredo MLF. A mulher idosa e a educação em saúde: saberes e praticas para promoção do envelhecimento saudável [tese]. Rio de Janeiro (RJ): Escola de Enfermagem Anna Nery, Universidade Federal do Rio de Janeiro; 2005.

6. Lessa I. O adulto brasileiro e as doenças da modernidade: epidemiologia das doenças crônicas transmissíveis. Rio de Janeiro (RJ): Hucitec; 1998.

7. Diogo MID, Paschoal SMP, Cintra FA. Avaliação global do idoso In: Duarte YAO, Diogo MJD. Atendimento domiciliar: um enfoque gerontológico. São Paulo (SP): Atheneu; 2000. p. 145-7|.

8. Diogo MJDE, Kawasaki K Assistência domiciliaria ao idoso: perfil do cuidador formal - parte II. Rev Esc Enferm USP 200I; 35(4): 320-7.
9. North American Nursing Diagnosis Association. Diagnósticos de enfermagem da NANDA:: definições e classificação 200 I2002. Porto Alegre (RS): Artmed; 2002.

10. Sakano LM, Yoshitome AY. Diagnósticos e intervenções de enfermagem em idosos hospitalizados. Acta Paul Enferm 2007; 20(4): 495-8

11. Chizzotti, A. Pesquisa em ciências humanas e sociais. São Paulo (SP): Cortez; 1998.

12. Ministério da Saúde (BR). Conselho Nacional de Saúde. Resolução no. 196, de 10 de outubro de 1996. Dispõe sobre diretrizes e normas regulamentadoras de pesQuisas envolvendo seres humanos. Bioética 1996; 4(Supl 2): 15-25.

13. Marconi MA, Lakatos EM. Técnicas de pesquisa: planejamento e execução de pesquisas, amostragens e técnicas de pesQuisas, elaboração, análise e interpretação de dados. São Paulo (SP): Atlas; 1999.

14. Paschoal SMP. Autonomia e independência. In: Papaléo Netto M. Gerontologia. São Paulo (SP): Atheneu; 1996. p. 3 I 3-23.

15. Carpenito LJ. Diagnóstico de enfermagem: aplicação à prática clínica. 10ª ed. Porto Alegre (RS): Artes Médicas; 2005.

16. Silva FAB, Batista MAS. A consulta de enfermagem ao idosoaspectos relevantes. Programa Saúde da Família. Santo Antonio do Descoberto (GO); 2005.

17. Ministério da Saúde (BR). Portaria ${ }^{0} 2.529$ de 19 de Outubro de 2006. A portaria institui a internação domiciliar no âmbito do SUS. Brasília (DF): Ministério da Saúde; 2006. 